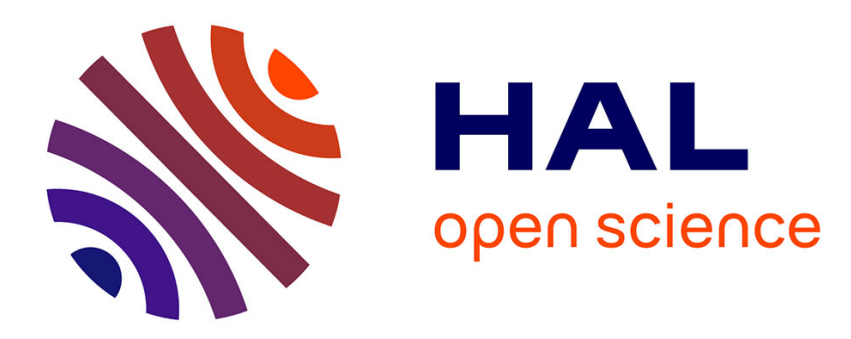

\title{
SPIN FLUCTUATIONS IN ITINERANT ELECTRON MAGNETISM
}

\author{
T. Moriya, Y. Takahashi
}

\section{To cite this version:}

T. Moriya, Y. Takahashi. SPIN FLUCTUATIONS IN ITINERANT ELECTRON MAGNETISM. Journal de Physique Colloques, 1978, 39 (C6), pp.C6-1466-C6-1471. 10.1051/jphyscol:19786588 . jpa-00218080

\section{HAL Id: jpa-00218080 https://hal.science/jpa-00218080}

Submitted on 1 Jan 1978

HAL is a multi-disciplinary open access archive for the deposit and dissemination of scientific research documents, whether they are published or not. The documents may come from teaching and research institutions in France or abroad, or from public or private research centers.
L'archive ouverte pluridisciplinaire HAL, est destinée au dépôt et à la diffusion de documents scientifiques de niveau recherche, publiés ou non, émanant des établissements d'enseignement et de recherche français ou étrangers, des laboratoires publics ou privés. 


\title{
SPIN FLUCTUATIONS IN ITINERANT ELECTRON MAGNETISM
}

\author{
T. Moriya and $Y$. Takahashi \\ Institute for Solid State Physies, University of Tokyo, Roppongi, Tokyo, Japan
}

Résumé.- On expose une théorie générale des fluctuations de spins et les propriétés thermodynamiques du magnétisme électronique itinérant, interpolation entre les limites faible et forte du ferromagnétisme. On donne une expression unifiée de la température de Curie et on discute la signification physique de la susceptibilité magnétique statique de Curie-Weiss. En tant que phénomènes nouveaux découlant de cette théorie, on discute les moments magnétiques locaux dépendant de la température comme ceux observés dans $\mathrm{CoS}_{2}, \mathrm{CoSe}_{2}$, etc. et les propriétés magnétiques et thermiques des semiconducteurs presque magnétiques connus de $\mathrm{Si}$.

Abstract.- A general theory of spin fluctuations and thermodynamical properties of itinerant electron magnets is developed, interpolating between the weakly and strongly ferromagnetic limits. A unified expression is given for the Curie temperature and the physical meaning of the Curie-Weiss magnetic susceptibility is discussed. As new phenomena derived from this theory the temperature-induced local magnetic moments as observed in $\operatorname{CoS}_{2}, \operatorname{CoSe}_{2}$, etc. and peculiar magnetic and thermal properties of nearly ferromagnetic semiconductors such as FeSi are discussed.

1. INTRODUCTION,- Ferro- and antiferromagnetism of transition metals has been one of the most controversial subjects in solid state physics for half a century. A well-known issue in this subject has been to choose between the itinerant and the localized models, and the meaning of the issue itself has been changing from time to time. Although the early version of it : "if the d electrons are itinerant or localized", seems to have been settled (the former is the case) in 1960 's, a long=standing question as to the existence or non-existence of the local magnetic moments in $d$ metals has been a serious one surviving even at present $/ 1 /$.

The difficulty of the problem arises from the fact that it is essential for the theory to go beyond the Hartree-Fock (HF)-random phase approximation (RPA), taking good account of the electron correlation in narrow d-bands particularly at finite temperatures. This problem, we believe, is best approached by studying the nature of spin and charge density fluctuations, particularly the former, from a general point of view as will be discussed in this note. As a matter of fact it seems there has been a substantial progress along this direction in recent years and the local moment picture is now regarded as a limiting form of more general spin density fluctuations.

In the opposite limit to the local moment case, where the spin fluctuations behave just like a set of mutually interacting local moments, we have, weakly ferro- and antiferromagnetic metals, where the spin fluctuation components in a small part of the q-space play a predominant role. The approach from this limit emphasizes the local nature in the reciprocal space of spin fluctuations and is naturally an extension of the HF-RPA theory to include the coupling among different $q$-components of spin density fluctuations.

The self-consistent renormalization (SCR) theory of spin fluctuations, which takes account of the renormalization effects of the spin fluctuations and the equilibrium state at the same time in a self=consistent fashion, has been presented several years ago $/ 2 /$. This line of approach has been remarkably successful not only in resolving classical difficulties associated with the HF-RPA theory, particularly those associated with the Curie-Weiss (CW) magnetic susceptibility, but also in explaining and predicting a number of qualitatively new features of the physical properties associated with the spin fluctuations, such as specific heat, NMR relaxation rate, electrical and thermal resistivities, and magneto-resistance, etc.

This approach, however, is justified only in the limit of small fraction of relevant part of the q-space and small amplitude of the spin density. In order to get a unified view of itinerant electron magnetism it is essential to go beyond this restriction and to interpolate between the abovementioned two limits, since there are great many magnetic metals ranging almost continuously between these two limits. 
Recently we have presented a rather simple spin fluctuation theory of ferromagnetic metals which is applicable to a general amplitude of the spin density and given a unified view to this problem, interpolating between the above mentioned two limits /3/. In the course of this investigation it was also found that in certain cases the amplitude of the local spin density fluctuations can be strongly temperature dependent and saturated at certain temperatures, leading to the local moment case. In other words we can have temperature-induced local magnetic moments $/ 4 /$.

In this note we discuss these latest developments focussing attention mainly to the CW susceptibility which has been one of the main origins of the above-mentioned controversy for 50 years.

\section{CURIE-WEISS MAGNETIC SUSCEPTIBILITY AND SPIN} FLUCTUATIONS.- The magnetic susceptibilities above the Curie temperature $\mathrm{T}_{\mathrm{C}}$ of all the ferromagnets obey the Curie-Weiss 1aw at least approximately. This fact has been the main reason why the local moment picture has been maintened for ferromagnetic metals, since only this picture was able to give a general explanation for the CW law before the SCR theory has appeared.

As a matter of fact, inadequacy of the local moment picture in giving a general explanation for the CW law of ferromagnetic metals has been pointed out earlier by Rhodes and Wohlfarth /5/. They estimated the effective local moment $\mathrm{p}_{\mathrm{C}}$ for various ferromagnetic metals from their Curie constants assuming the existence of the local moments and compared them with the saturation moments $\mathrm{p}_{\mathrm{s}}$ per atom at $0 \mathrm{~K}$. They found that $\mathrm{p}_{\mathrm{C}} / \mathrm{p}_{\mathrm{S}}$ is generally larger than 1 and can be quite large in some cases, particularly in weakly ferromagnetic metals. This is inconsistent with the local moment model which always gives $\mathrm{p}_{\mathrm{C}} / \mathrm{p}_{\mathrm{s}}=1$. They tried to explain this fact with the use of the Stoner theory, assuming very small effective Fermi energies, without general success.

This problem has been solved partially by the SCR theory $/ 2 /$, which in the weakly ferromagnetic limit gives the Curie constant much larger than is deduced from the saturation moment, assuming the local moment model, and is not even related with the latter. However, there are many ferromagnetic metals in the intermediate range and a plot of $\mathrm{p}_{\mathrm{C}} / \mathrm{p}_{\mathrm{s}}$ vs. $\mathrm{T}_{\mathrm{C}}$ gives almost continuous distribu- tion of this ratio with the lower bound of $1 / 6 /$. This indicates that the nature of spin fluctuations changes continuously form one limit to the other. Thus it is highly desired to have a theory of interpolation, which we will discuss in the following sections.

3. MODEL AND APPROXIMATIONS.- We consider the Hubbard-type Hamiltonian with the intra-atomic electron-electron interaction only and express the interaction Hamiltoninan in an isotropic form with respect to the spin density as follows :

$\mathrm{H}_{\mathrm{I}}=$ const. $-\mathrm{U} \sum_{\mathbf{j}} \overrightarrow{\mathrm{S}}_{\mathbf{j}}^{2}$

The free energy $F$ of the system is given by the following functional integral /7/ :

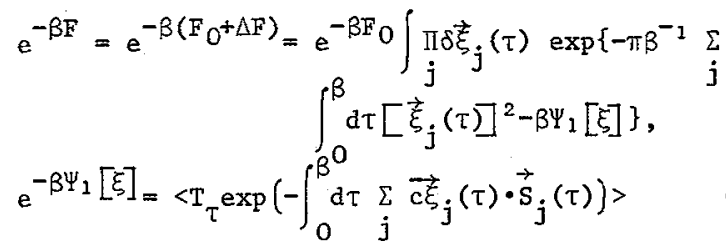

with

$\vec{s}_{j}(\tau)=e^{\tau H_{0}} \vec{S}_{j} e^{-\tau H_{0}}$,

$\bar{c}=(4 \pi U / \beta)^{1 / 2}$,

$\langle\mathrm{A}\rangle=\operatorname{Tr}\left[\mathrm{A} \exp B\left(\mathrm{~F}_{0}-\mathrm{H}_{0}\right)\right]$,

where $\mathrm{H}_{\mathrm{O}}$ and $\mathrm{F}_{\mathrm{O}}$ are the Hamiltonian and the free energy for $H_{I}=0$. According to this formalism the problem is reduced to calculating $\Psi_{1}[\xi]$, or studying the motion of the spin density of non-interacting electron system under a magnetic field $\vec{c} \vec{\xi}_{j}(\tau)$ which varies arbitrarily in space and time. The average spin density and the dynamical susceptibility are evaluated as follows :

$$
\begin{aligned}
&\left\langle\vec{\xi}_{j}(\tau)>=(\pi / U \beta)^{1 / 2}<\vec{\xi}_{j}(\tau)>\right. \\
& x_{q m}=x\left(q, i \omega_{m}\right)=(\pi / I)\left[\left\langle\vec{\xi}_{q m} \vec{\xi}_{q-m}>-<\vec{\xi}_{q m}><\vec{\xi}_{-q-m}>\right.\right. \\
&-(1 / 2 \pi)]
\end{aligned}
$$

$\vec{\xi}_{\text {qm }}^{\text {with }}=N_{0}^{-1 / 2} \beta^{-1} \Sigma_{j} \int_{0}^{\beta} \mathrm{d} \tau \vec{\xi}_{j}(\tau) \exp \left[\vec{i}\left(\vec{q} \cdot \vec{R}_{j}+\omega_{m} \tau\right)\right]$

where $I=U / N_{0}, N_{0}$ being the number of atoms in the crystal and the average is taken over $\xi$ with the weight factor given in equation (3.2). In the above and throughout this paper the magnetic moment and the susceptibility are given in units of $g \mu_{B}$ and $\mathrm{g}^{2}{\mu_{B}}^{2}$, respectively, where $g$ is the $g$-factor and $\mu_{B}$ the Bohr magneton.

We first expand $\Psi_{1}[\xi]$ in terms of $\xi$ and take only the second order terms. We have

$\Psi_{1}^{(2)}[\xi]=-\left(c^{2} / 2\right) \sum_{\mathrm{q} m} \sum \chi_{\mathrm{qm}}^{0} \vec{\xi}_{\mathrm{qm}} \cdot \vec{\xi}_{-\mathrm{q}-\mathrm{m}}$, 
with

$c=(4 \pi I / \beta)^{\sqrt{2}}$

where $\chi_{\mathrm{qm}}{ }^{0}$ is the dynamical susceptibility for $I=0$. The use of this expression for $\Psi_{1}[\xi]$ leads to the RPA dynamical susceptibility. The higher order terms are generally hard to evaluate without making some approximations. One possible way is to use a Hartree-type approximation for $\Psi_{1}[\xi]$, which in general is given by $/ 8 /$

$\Phi_{1}[\xi]=-\left(c^{2} / 2\right) \sum_{\mathrm{q} m}^{\sum} \bar{\chi}_{\mathrm{qm}}>\vec{\xi}_{\mathrm{qm}} \cdot \vec{\xi}_{-\mathrm{q}-\mathrm{m}}$

where $\bar{\chi}_{\mathrm{qm}}$ is the dynamical susceptibility for vanishing $I$ under the fluctuating field $c \xi$. The average is taken with an approximate weight factor

where $\Psi_{1}$ is substituted by $\Phi_{1}$.

It is convenient at this stage to introduce a model functional for $\Psi_{1}[\xi]$. We introduce the average square amplitude of the local field

$\mathrm{x}_{\mathrm{m}}=\frac{1}{\mathrm{~N}_{\mathrm{O}} \beta} \sum_{\mathrm{q}} \vec{\xi}_{\mathrm{qm}} \cdot \vec{\xi}_{-\mathrm{q}-\mathrm{m}}$

which is related with the average square amplitude of the local spin density by the following relation :

$\left\langle\overrightarrow{\mathrm{S}}_{\mathrm{j}}^{2}\right\rangle=\frac{\pi}{\mathrm{U}} \sum_{\mathrm{m}}\left(\mathrm{x}_{\mathrm{m}}-\frac{3}{2 \pi \beta}\right)$,

We assume that the coupling among the spin fluctuation modes is local and the higher order terms in $\Psi_{1}[\xi]$ are mainly expressed in terms of $\mathrm{x}_{\mathrm{m}}$. Our model functional is

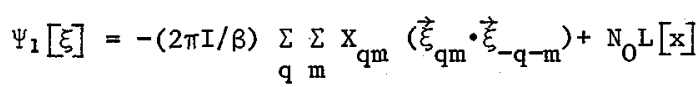

with

$\mathrm{x}_{\mathrm{qm}}=\bar{\chi}_{\mathrm{qm}}-\mathrm{N}_{\mathrm{o}}^{-1} \Sigma \bar{\chi}_{\mathrm{qm}}$

where $\bar{\chi}_{\mathrm{qm}}$ is now regarded as a function of $\mathrm{x}$ only. This means that among the higher order terms related with the relative orientation of the local field $\vec{\xi}_{j}$ and $\vec{\xi}_{\ell}$ we leave only those linear in $\left(\vec{\xi}_{j m} \cdot \vec{\xi}_{l-m}^{j}\right)$. The model functional of this form may be calculated with the use of various approximation methods. We leave this for later discussion.

An important point here with this model functional is that it represents both weakly ferromagnetic and local moment cases. When $\xi$ or $x$ is small as in weakly ferro- and antiferromagnetic metals, $\bar{\chi}_{\mathrm{qm}} \bar{\chi}_{.00}$ may well be approximated by $\chi_{\mathrm{qm}}{ }^{0} / \chi_{00}{ }^{0}$, and we may use a long wave approximation expanding $L[x]$ up to the second order. This leads to the SCR theory presented previously, if we work out the functional integral. On the other hand, a local moment-like case may be expected when $\Sigma x_{m}+L[x]$ has a deep minimum at fairly large values of $x$ and $x_{q m}$ is relatively small. Furthermore we will encounter the local moment case when the amplitude of the 10cal spin fluctuation is saturated as will be discussed shortly. Thus we may expect that this model functional can not only describe the above mentioned two limits but also provide us with an interpolation between these limits, leading to a general qualitative account of itinerant electron ferromagnetism.

Now the free energy is given by

$$
\begin{aligned}
& e^{-\beta \Delta F}=\int_{m} \pi d x_{m} \exp \left\{-\pi \beta N_{0}\left(\sum x_{m}+L[x]\right)-\beta F_{1}[x]\right\} \\
& \mathrm{e}^{-\beta \mathrm{F}_{1}[\mathrm{x}]}=\int \delta \xi \exp \left[2 \pi I \underset{\mathrm{q}}{\Sigma} \sum_{\mathrm{m}} \mathrm{x}_{\mathrm{qm}} \vec{\xi}_{\mathrm{qm}} \cdot \vec{\xi}_{-\mathrm{q}-\mathrm{m}}\right] \\
& \text { II } \delta\left[\mathrm{x}_{\mathrm{m}}-\left(\mathrm{N}_{0} \beta\right)^{-1} \sum_{\mathrm{q}} \vec{\xi}_{\mathrm{qm}} \cdot \vec{\xi}_{-\mathrm{q}-\mathrm{m}}\right]
\end{aligned}
$$

$\mathrm{F}_{1}[\mathrm{x}]$ can be evaluated by using the Lagrange multipliers as follows :

$\mathrm{F}_{1}[\mathrm{x}]=(3 / 2 \beta) \sum_{\mathrm{q}} \sum \log \left[2 \mathrm{I}\left(\lambda_{\mathrm{m}}-\mathrm{X}_{\mathrm{qm}}\right)\right]-2 \pi U \sum_{\mathrm{m}} \lambda_{\mathrm{m}} \mathrm{x}_{\mathrm{m}}$

with

$\mathrm{x}_{\mathrm{m}}=(3 / 4 \pi \mathrm{U}) \sum_{\mathrm{q}} \sum_{\mathrm{m}}\left(\lambda_{\mathrm{m}}-\mathrm{x}_{\mathrm{qm}}\right)^{-1}$

Evaluating the integral in equation (3.10) with the saddle point approximation, we finally get the following results for the susceptibility :

$\chi_{\mathrm{qm}}=\bar{\chi}_{\mathrm{qm}} /\left(1-2 \overline{\mathrm{I}}_{\mathrm{qm}}\right)$,

$\bar{X}_{\mathrm{qm}}=\mathrm{X}_{\mathrm{qm}}-\left(\partial \mathrm{L} / \partial \mathrm{x}_{\mathrm{m}}\right) / 2 \mathrm{I}$.

$x_{m}=\left(3 / 2 \pi N_{0} \beta\right) \sum\left(1-2 I \bar{\chi}_{q m}\right)^{-1}$

Here we point out that the amplitude of the local spin density is bounded and therefore the following relation as derived from equation (3.7) must be satisfied

$\sum \sum_{\mathrm{m}} \mathrm{X}_{\mathrm{qm}} \leq \frac{1}{3} \mathrm{~N}_{\mathrm{O}}^{2} \mathrm{BM}_{\mathrm{C}}^{2}$

where $M_{c}{ }^{2}$ is determined by the number of electrons per atom and the strength of electron correlation. We note that this relation should automatically be satisfied if we calculate $L[x]$ appropriately.

For later convenience we rewrite equation (3.13) as follows :

$\chi_{\mathrm{qm}}=\frac{1}{2 I} \frac{\mathrm{f}_{\mathrm{qm}}}{\delta+1-\mathrm{f}_{\mathrm{qm}}}, \quad \mathrm{f}_{\mathrm{qm}}=\bar{\chi}_{\mathrm{qm}} / \bar{\chi}_{\mathrm{OO}}$

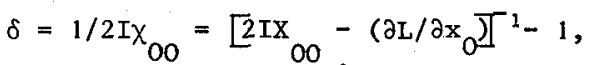

$x_{m}(\delta, T)=\frac{3}{2 \pi N_{0} \beta} \quad \sum \frac{\delta+1}{\delta+1-f_{q m}}$

When $f_{q m}, x_{O O}$ and $L[x]$ are given we can solve these equations for $\delta$. At this point we make a simpli- 
fying approximation of assuming $\partial \mathrm{L} / \partial \mathrm{x}_{0}$ to be a function of $x_{0}$ only. This approximation may be good at high temperatures. We have

$\delta=\left[2 \mathrm{IX}_{\mathrm{OO}}-\mathrm{L}^{\prime}\left(\mathrm{x}_{\mathrm{O}}\right)\right]^{-1}-1$

$\mathrm{x}_{0}(\delta, \mathrm{T})=\frac{3}{2 \pi \beta}\left\langle\frac{\delta+1}{\delta+\sigma}\right\rangle, \quad \sigma=1-\mathrm{f}_{\mathrm{qO}}$

where the average is taken over $q$. The Curie temperature is given by

$1-2 \mathrm{IX}_{00}+\mathrm{L}^{\prime}\left(\mathrm{x}_{\mathrm{C}}\right)=0$,

$\left.x_{C}=\left(3 k_{B} T_{C} / 2 \pi\right)<1 / \sigma\right\rangle$,

and the magnetic susceptibility above $\mathrm{T}_{\mathrm{C}}$ is given by

$1 / X=2 I \delta=2 I \gamma\left(x_{0}-x_{C}\right) /\left[1-\gamma\left(x_{0}-x_{C}\right)\right]$

with

$\gamma=L^{\prime \prime}\left(\mathrm{x}_{\mathrm{C}}\right)-2 \mathrm{I}\left[\bar{\partial} \mathrm{x}_{\mathrm{OOO}} / \partial \mathrm{x}_{0}\right]_{\mathrm{x}_{\mathrm{O}}}=\mathrm{x}_{\mathrm{C}}$

$L^{\prime \prime}\left(x_{0}\right)$ is the longitudinal stiffness constant for the local spin density fluctuation or the stiffness constant for the change in amplitude of the local spin density and is the main part of $\gamma$.

\section{CURIE TEMPERATURE AND MAGNETIC SUSCEPTIBILITY} (STANDARD CASE).- Curie temperature is given by equation (3.17) where the value of $x_{C}$ may be related with the saturation moment at $\mathrm{T}=0 \mathrm{~K}$. In the present approximation on $1 y \xi_{00}$ has a finite value at $\mathrm{T}=0$, thus we have $\mathrm{x}_{\mathrm{O}}=\xi_{00}{ }^{2} / \mathrm{N}_{0} \beta$. Minimizing $\Psi[\xi]$ with respect to $x_{0}$ we get $1-2 I x_{00}-L^{\prime}\left(x_{0}\right)=0$. Therefore if we simply neglect the T-dependence of $L\left(x_{0}\right)$ we get $x_{C}=x_{0}=U_{0}{ }^{2} / \pi$, where $M_{0}$ is the saturation moment per atom. A more detailed consideration gives rise to a numerical factor $\eta$ of order 1 and we have $/ 3 /$

$\mathrm{T}_{\mathrm{C}}=2 \operatorname{num}_{\mathrm{O}}{ }^{2} / 3 \mathrm{k}_{\mathrm{B}}<1 / \sigma>$

We note that this expression in the weakly ferromagnetic limit agrees with the result of static approximation in the SCR theory. It is also possible to reproduce the SCR result with the dynamical effects, making proper approximations in equation (3.15). Also, if we make the following replacement: $2 \mathrm{Ux}_{\mathrm{qO}} / \bar{\chi}_{\mathrm{OO}} \mathrm{N}_{\mathrm{O}}=\mathrm{J}(\mathrm{q})$

equation (4.1) leads to the result for the Heisenberg model with the Fourier transform of the interatomic exchange interaction constant given by $\mathrm{J}(\mathrm{q})$. Therefore, equation (4.1) seems to give an interpolation between the two limits.

Magnetic susceptibility is given by the following equation dedeuced from equations (3.16 - 18)
$1 / X=2 I \delta$

$\frac{\delta}{1+\delta}=\frac{T}{T_{0}}\left\langle\frac{\delta+1}{\delta+\sigma}>-\frac{T_{C}}{T_{0}}\left\langle\frac{1}{\sigma}\right\rangle\right.$

with

$\mathrm{k}_{\mathrm{B}} \mathrm{T}_{\mathrm{O}}=2 \pi / 3 \gamma$

This equation can be solved if we give a distribution function $P(\sigma)$ for $\sigma$. We first discuss two 1imiting cases.

When $\mathrm{T}_{0} / \mathrm{T}<1 / 0>$ is large $\delta$ is generally small and we get an approximate expression

$X=C_{\ell} /\left(T-T_{C}\right) ; \quad C_{\ell}=T_{0} / 2 \mathrm{I}<1 / \sigma>$

In this case the Curie constant is inversely proportional to the longitudinal stiffness constant $\gamma$, indicating the importance of the longitudinal local spin fluctuations.

When $\mathrm{T}_{0} / \mathrm{T}_{\mathrm{C}}<1 / \sigma>$ is sma11 we get an approximate Curie-Weiss law with the Curie constant given by

$\mathrm{C}_{\mathrm{t}}=\mathrm{T}_{\mathrm{C}}<1 / \sigma>/ 2 \mathrm{I}=\mathrm{nN}_{\mathrm{O}} \mathrm{M}_{\mathrm{O}}{ }^{2} / 3 \mathrm{k}_{\mathrm{B}}$

We see that the situation is just as in the local moment model. This is natural since small $\mathrm{T}_{0}$ means large longitudinal stiffness. The Weiss constant in this case agrees with $\mathrm{T}_{\mathrm{C}}$ only approximately.

In the intermediate case the Curie constant is very roughly given by the sum of the above two terms, $C_{\ell}+c_{t}$, though the Curie-Weiss law itself has smaller range of validity.

It is interesting to stress that the $\mathrm{CW}$ law is best satisfied in the above-mentioned two opposite limits and the linearity of $1 / X-T$ curve becomes worse in the intermediate range. This is qualitatively consistent with the experimental results /9/. For further details including a numerical example with the use of the distribution function : $P(\sigma)=\left(2 / \pi a^{2}\right)[\sigma(2 a-\sigma)]^{\sqrt{2}}$

we refer to reference $/ 3 /$.

In the present theory we use a small number of physical parameters in giving a unified description of itinerant electron ferromagnets. We have $M_{0}, T_{0}$ or $\dot{\gamma},\langle 1 / \sigma\rangle$, and $I$ as independent parameters. Some analyses of existing experimental data in terms of these parameters are given in reference $13 /$. We only note here that in general both the transversal and longitudinal components of the spin fluctuations are important. The latter is predominant in the weakly ferromagnetic limit and the former dominates in the local moment limit. Naturally in the case of magnetic insulators we have only the former and the problem is much simpler 
than in the present case.

The present model of local mode-mode coupling is expected to cover fairly large part of itinerant electron magnets. Although there are examples which require some modifications of the model $/ 10 /$, spin fluctuations of large amplitude in general may be treated in the same line as the present approach.

5. TEMPERATURE-INDUCED LOCAL MAGNETIC MOMENTS.- In this section we consider the cases where the amplitude of the local spin fluctuation is strongly temperature dependent, as is expected when the longitudinal stiffness constant $\gamma$ is sma11 or equivalently the coupling among the spin fluctuation modes is weak. In such a case the amplitude of the local spin fluctuations increases with temperature as may be seen from equation (3.16). This increase, however, is limited by the saturation condition of equation (3.14) which may be rewritten in the static approximation as

$\frac{3 \mathrm{k}_{\mathrm{B}} \mathrm{T}}{2 \mathrm{U}}<\frac{1-\sigma}{\delta+\sigma}>=\mathrm{M}_{\mathrm{C}}^{2}$

The temperature of saturation $T^{\mathbf{*}}$ may be estimated from equations $(3.16)$ and $(5.1)$. We have

$3 k_{B} T^{*}<\frac{1-\sigma}{1-\alpha+\alpha \sigma}>=M_{C}{ }^{2} N_{O} / \bar{x}_{0 O}$

with

$\alpha=2 I \bar{\chi}_{00}$

Estimating $\mathrm{N}_{\mathrm{O}} / \overline{\mathrm{X}}_{0 O}$ very roughly as the band width $\mathrm{w}$, we get

$T^{\mathbf{x}} \simeq(1-\alpha+\alpha<\sigma>) \mathrm{WM}_{\mathrm{C}}{ }^{2} / 3 \mathrm{k}_{\mathrm{B}}$

$\mathrm{T}^{\text {F }}$ may easily be in an accessible range. Below $\mathrm{T}^{*}$ the susceptibility is given by equation (3.16) and above $T^{\text {* }}$ it is given by equation $(5.1)$ and $X=1 / 2 I \delta$. It may clearly be seen from equation (5.1) that above $T^{*}$ we have a Curie-Weiss susceptibility of local mome- type when $\sigma$ is not large.

This type of behavior has been observed in $\mathrm{Co}\left(\mathrm{S}_{\mathrm{x}} \mathrm{Se}_{1-\mathrm{x}}\right)_{2}$ system with the pyrite-type crystal structure where we have one electron per co atom in the two-fold degenerate $e_{g}$ bands. $\mathrm{CoS}_{2}$ is a ferromagnetic metal with $\mathrm{T}_{\mathrm{C}}=120 \mathrm{~K} .1 / \mathrm{X}-\mathrm{T}$ plot above $T_{C}$ shows a break at around $T^{\mathbf{z}} \simeq 400 \mathrm{~K}$ and the slope above $\mathrm{T}^{\mathbf{x}}$ corresponds to the local moment of $1 \mu_{B}$ per Co atom, while the slope below $T^{m}$ is sma1ler, indicating a significant contribution of the longitudinal spin fluctuations to the Curie constant below $T^{*}$. When we substitute $S$ with Se both $\mathrm{T}_{\mathrm{C}}$ and $\mathrm{T}^{\mathrm{F}}$ are lowered, the decrease of the latter being much slower. This local moment-type CW sus- ceptibility above $\mathrm{T}^{*}$ persists even beyond the critical concentration for ferromagnetism $(x<0.88)$. In this case we have exchange-enhanced Pauli paramagnetism of nearly ferro- or antiferromagnetic metals below $\mathrm{T}^{*}$, and the appearance of the temperatureinduced local moments is most dramatic.

It may also be possible to interpret the magnetic and thermal properties of $\mathrm{V}_{2} \mathrm{O}_{3}$ under pressure and those of FeSi, a nearly ferromagnetic semiconductor, in terms of the present mechanism. The latter will be discussed in the next section.

This phenomenon takes place when the Fermi level is not far from the band edge and the longitudinal stiffness constant $L^{\prime \prime}\left(x_{0}\right)$ and the width of the distribution $P(\sigma)$ of $\sigma$, i.e., $\langle 1 / \sigma\rangle^{-1}$ is small. The second condition may be satisfied when the effective local density of states curve is fairly flat, having no conspicuous peak structure. The third condition is expected to be satisfied when the band is nearly quarter-filled just as in $\mathrm{Co}\left(\mathrm{S}_{\mathrm{x}} \mathrm{Se}_{1-\mathrm{x}}\right)_{2}$, or quarterempty. For further details we refer to reference 14/. Finally we point out that high temperature properties of $\mathrm{Ni}, \mathrm{Pd}$ and $\mathrm{Pt}$ may also be explained in terms of this mechanism in an approximate sense.

6. NEARLY FERROMAGNETIC SEMICONDUCTORS.- We now note that the present theory as applied to nearly ferromagnetic semiconductors seems to explain peculiar magnetic and thermal properties of FeSi which have been an enigma for a long time.

The magnetic susceptibility of FeSi is very small at low temperatures and starts to increase exponentially at around $100 \mathrm{~K}$. After having a broad maximum at around $500 \mathrm{~K}$ it becomes $\mathrm{CW}$-type corresponding to $2 \mu_{B}$ per Fe at high temperatures. The electronic specific heat has an anomalous peak at around $200 \mathrm{~K}$. Jaccarino et al. /11/ tried to explain these phenomena with the use of a seminconductor model within the one-electron approximation. In order to obtain a good fit, however, they had to assume vanishing band widths both for the valence and the conduction bands. This is quite unreasonable.

The story is changed dramatically when we take account of the effect of spin fluctuations following the present theory. We consider a simple semiconductor with the local density of states given by $\rho(\varepsilon)= \begin{cases}\rho, & \text { for } \Delta<|\varepsilon|<\Delta d \\ 0, & \text { otherwise, }\end{cases}$

with $2 \rho(d-1)=N_{0}$ and the Fermi level at $\varepsilon=0$. We then obtain 
$\begin{aligned} L^{\prime}\left(x_{0}\right)= & \frac{I \rho t}{2 b} \log \left\{\left[1+\cosh \frac{d+b}{t}\right]\left[1+\cosh \frac{1-b}{t}\right] /\right. \\ & {\left.\left[1+\cosh \frac{d-b}{t}\right]\left[1+\cosh \frac{1+b}{t}\right]\right\} }\end{aligned}$

with

$\mathrm{b}=\left(\pi \mathrm{Ux}_{0}\right)^{1 / 2} / \Delta$

$\mathrm{t}=\mathrm{k}_{\mathrm{B}} \mathrm{T} / \Delta$

The magnetic susceptibility calculated for $\mathrm{d}=10$,

and the distribution function (4.7) with $a=0.1$ are shown in figure 1 for a few values of Ip. Conside-

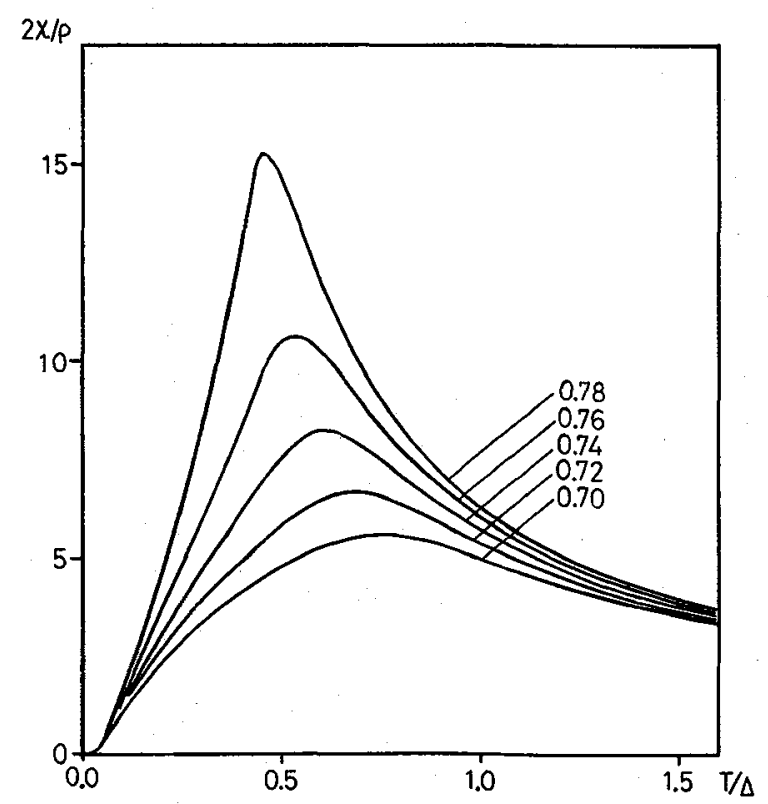

Fig. 1 : Magnetic susceptibility of a nearly ferromagnetic semiconductor for various values of the exchange interaction $I$; numerical value for $I / I_{c}$ $I_{c}$ being the critical value for ferromagnetism, is shown for each line, $\Delta$ is half the band gap energy.

ring that the local spin fluctuation is saturated in the high temperature side of the maximum we may expect a peak in the specific heat significantly below the maximum of the susceptibility. Calculation of the specific heat is now under way and preliminary results seem to be consistent with the above expectation. This simple calculation seems to give a good qualitative explanation of the properties of FeSi and to provide us with yet another example showing the real importance of the correlated modes of spin fluctuations.

\section{References}

/1/ For a review of theoretical developments in early stages see for example : Herring,G., Magnetism IV, ed. Rado,G.T. and Suhl,H., (Academic Press) 1966

12/ For a review see : Moriya,T., Physica 86-88B (1977) 356, 91B (1977) 235

/3/ Moriya,T. and Takahashi,Y., J. Phys. Soc. Japan 45 (1978) to be published

/4/ Moriya,T., Solid State Commun. 26 (1978) 483

/5/ Rhodes,P.R. and Wohlfarth,E.P., Proc. Roy. Soc. (London) 273 (1963) 247

16/ Kawarazaki,S., Yasuoka,H., Nakamura,Y. and Wernick,J.H., J. Phys. Soc. Japan 41 (1976) 1171

17/ Evenson,W.E., Schrieffer,J.R. and Wang,S.Q., J. App 1. Phys. 41 (1970) 1199

18/ Hertz,J.A, and Klenin,M.A., Phys, Rev, B10 (1974) 1084 ; Physica 91B (1977) 49

19/ Ogawa,s., Researches of the Electrotechnical Laboratory No. 735 (1972)

/10/ Moriya,R. and Usami,k., Solid State Commun. 23 (1977) 935

/11/ Jaccarino,V., Wertheim,G.K., Wernick,J.H., Walker,L.R. and Arajs,S., Phys. Rev. 160 (1967) 476 